\title{
Étude des conditions optimales permettant la pycniogenèse de Phaeosphaeria (Leptosphaeria) nodorum (MülI) Hedj agent de la septoriose du blé
}

\author{
P Halama 1, L Lacoste 2 \\ 1 Institut supérieur d'agriculture, 41, rue du port, 59046 Lille cedex \\ ${ }^{2}$ Muséum national d'histoire naturelle, laboratoire de cryptogamie, 12 rue Buffon, 75005 Paris, France
}

(Reçu le 26 août 1991; accepté le 3 août 1992)

\begin{abstract}
Résumé - La pycniogénèse de $P$ nodorum peut être obtenue sur de nombreux milieux de culture, mais une perte des potentialités conidiogènes est toujours observée lors du maintien des souches en conditions de laboratoire, particulièrement dans le cas de souches issues d'ascospores. Un milieu synthétique contenant $4000 \mathrm{mg} / \mathrm{l}$ de carbone et $400 \mathrm{mg} / \mathrm{l}$ d'azote respectivement sous forme de galactose et de glycine permet une bonne production de pycnides et de cirrhes. Ces concentrations donnent un rapport $\mathrm{C} / \mathrm{N}=10$ qui apparaît le plus favorable à la pycniogénèse. La comparaison de la pycniogénèse pour 2 souches mono-ascosporées conduit à la définition des conditions optimales de température $\left(14^{\circ} \mathrm{C}\right)$ et d'éclairement $(12 \mathrm{~h}$ en lumière noire). Vis-à-vis de l'éclairement énergétique, 1 des 2 souches manifeste pour la pycniogénèse un optimum plus large (400 et $600 \mu \mathrm{w} / \mathrm{cm}^{2}$ ) alors que l'autre souche a un optimum uniquement à $600 \mu \mathrm{w} / \mathrm{cm}^{2}$.
\end{abstract}

Phaeosphaeria nodorum / Leptosphaeria / pycniogénèse / milieu de culture / température / lumière

Summary - Optimal conditions for pycniogenesis in Phaeosphaeria (Leptosphaeria) nodorum, the causal agent of glume blotch disease. While, the sporulation of $\mathrm{P}$ nodorum is obtained on many media, a loss of sporulation is always observed during maintenance in laboratory conditions, in particular for cultures derived from single ascospores. A synthetic medium containing $4000 \mathrm{mg}$ carbon per I and $400 \mathrm{mg}$ nitrogen per I in the form of galactose and glycine respectively permits a high level of pycnidial formation and cirral production. These concentrations provide a ratio of $\mathrm{C} / \mathrm{N}=$ 10 which is the most favourable for sporulation. Comparison of sporulation between 2 strains resulted in the definition of optimal temperature $\left(14^{\circ} \mathrm{C}\right.$ ) and of light conditions (a 12-h photoperiod with near ultraviolet). The most favourable light intensity value for pycniogenesis of one strain was greater ( 400 or $600 \mu \mathrm{w} / \mathrm{cm}^{2}$ ) than for another strain for which the optimum intensity was only $600 \mu \mathrm{w} / \mathrm{cm}^{2}$.

Phaeosphaeria nodorum / Leptosphaeria / pycniogenesis / culture medium / temperature / light

\section{INTRODUCTION}

Bien que la pycniogénèse de Septoria nodorum, forme conidienne de Phaeosphaeria nodorum, puisse être obtenue sur différents milieux de culture dans le cas d'isolats fraîchement récoltés, de nombreux auteurs ont rapporté la difficulté à maintenir cette conidiogénèse dans le temps. D'autre part, des différences de vitesse de croissance et des variations morphologiques entre différents isolats de $S$ nodorum ont été obser- vées (Scharen et Krupinsky, 1970; Griffiths et Ao, 1980; Allingham et Jackson, 1981).

Lors d'un travail antérieur (Halama et Lacoste, 1991) sur des souches monoascosporées obtenues à partir de périthèces produits in vitro, nous avons mis en évidence des différences entre ces souches pour les critères suivants: aspects culturaux, croissance mycélienne, macro- et micropycniogénèse. II a été montré que le maintien des souches pendant plusieurs mois sur milieux naturels (pailles de blé, fragments de carottes) ou synthétiques (Richards, 1951) dans les condi- 
tions de laboratoire entraînait fréquemment une perte des facultés conidiogènes pour l'un des groupes de compatibilité sexuelle chez cette espèce hétérothallique.

Cette régression constitue un facteur limitant pour les études génétiques.

L'objet de cette étude est de préciser, d'une part, la composition d'un milieu synthétique et d'autre part, les conditions physiques permettant une conidiogénèse optimale et stabilisée.

\section{MATÉRIEL ET MÉTHODES}

\section{Souches utilisées}

À partir des périthèces produits in vitro (Halama et Lacoste, 1991), des isolements d'ascospores sont effectués à l'aide d'un micromanipulateur de De Fontbrune. Les ascospores isolées sont transférées dans de l'eau de pomme de terre $(10 \mathrm{~g}$ de pulpe/l d'eau, après ébullition pendant $5 \mathrm{~min}$, décantation pendant $1 \mathrm{~h}$ puis ajustement du filtrat à $\mathrm{pH} 4,5$ ). Les colonies mycéliennes développées à $18^{\circ} \mathrm{C}$, sont transférées après $24 \mathrm{~h}$ d'incubation en tubes de Roux sur des fragments de carotte stériles. Une souche mono-ascosporée (A 3.5) a été utilisée pour l'étude du rapport $C / N$ et 2 souches mono-ascosporées ont été retenues (A2.3 et A2.6) pour l'étude des facteurs physiques. Lorsque ces 2 souches sont croisées, des périthèces fertiles sont observés après $60 \mathrm{j}$ de culture. La confrontation de ces souches avec d'autres souches précédemment étudiées (séries $A 1$ et $\mathrm{B} 1$ ) permet de les classer dans 2 groupes de compatibilité sexuelle complémentaires : $+(A 2.3),($ A 3.5) et $-(A 2.6)$.

\section{Milieux de culture}

Trois types de milieux ont été utilisés : 2 milieux de référence (Richards, 1951 et Czapek-Dox V8, Cooke et Gareth-Jones, 1970) et un milieu dérivé du milieu de Morquer (1931), modifié par Lacoste (1965) puis Dehorter (1985). II diffère des 2 milieux de référence par un apport minéral plus étendu et plus équilibré. Sa composition, qui comporte les 5 macro-éléments classiques ( $K, P, S, M g$ et $\mathrm{Ca}$ ) et des microéléments ( $\mathrm{Zn}$, $\mathrm{Mn}, \mathrm{Mo}, \mathrm{Fe}$ et $\mathrm{Cu}$ ) rarement tous utilisés par les auteurs, est la suivante:

$\mathrm{KH}_{2} \mathrm{PO}_{4}, 800 \mathrm{mg} ; \mathrm{MgSO}_{4}, 7 \mathrm{H}_{2} \mathrm{O}, 250 \mathrm{mg}$; $\mathrm{NaH}_{2} \mathrm{PO}_{4}, \mathrm{H}_{2} \mathrm{O}, 100 \mathrm{mg} ; \mathrm{CaCl}_{2}, 50 \mathrm{mg} ; \mathrm{Zn} \mathrm{SO}$, $7 \mathrm{H}_{2} \mathrm{O}, 15 \mathrm{mg} ; \mathrm{FeSO}_{4}, 7 \mathrm{H}_{2} \mathrm{O}$-EDTA, $15 \mathrm{mg}-10 \mathrm{mg} ; \mathrm{Mn}$ $\mathrm{SO}_{4}, \mathrm{H}_{2} \mathrm{O}, 5 \mathrm{mg} ; \mathrm{BO}_{3} \mathrm{H}_{3}, 3 \mathrm{mg} ; \mathrm{Cu} \mathrm{SO} 4,5 \mathrm{H}_{2} \mathrm{O}, 1 \mathrm{mg}$; $\mathrm{MoNa}_{2} \mathrm{O}_{4}, \mathrm{H}_{2} \mathrm{O}, 1 \mathrm{mg}$. Ce milieu minéral est complété par un apport de vitamines : bichlorure de thiamine,
$0,2 \mathrm{mg}$; hydroxychlorure de pyridoxine, $0,2 \mathrm{mg}$; biotine (D+), 0,01 mg. La gélose est apportée à 1,5\%; eau distillée, $1000 \mathrm{ml}$; le pH est ajusté à 5,5.

Le carbone et l'azote sont fournis sous différentes formes et concentrations permettant de faire varier le rapport $\mathrm{C} / \mathrm{N}$.

Pour étudier la morphogénèse, des quantités variables de galactose $(1 ; 2,5 ; 5 ; 10 ; 15 ; 20$ et $25 \mathrm{~g} / \mathrm{l})$ et de glycine $(0,134 ; 0,269 ; 0,538 ; 1,075$ et $2,150 \mathrm{~g} / \mathrm{l})$ sont incorporées au milieu de base afin d'obtenir les concentrations finales suivantes:

- carbone : 400, $1000,2000,4000,6000,8000$ et $10000 \mathrm{mg} / \mathrm{l}$;

- azote : 25, 50, 100, 200, $400 \mathrm{mg} / \mathrm{l}$.

En fonction des associations galactose-glycine, les valeurs des rapports $\mathrm{C} / \mathrm{N}$ sont comprises entre 1 et 160.

Le milieu optimal retenu sera désigné sous le nom de milieu $\mathrm{S}$.

\section{Conditions de culture}

Après ensemencement par une suspension de pycniospores, étalée sur toute la surface du milieu de culture, les incubations sont réalisées à 4 températures $\left(10,14,18\right.$ et $\left.24^{\circ} \mathrm{C}\right)$, et sous un éclairement constitué de lumière de Wood $(300 \mathrm{~nm}<\lambda<400 \mathrm{~nm}$, tubes Sylvania Blacklight Blue $F 36$ et $F 20$ ) avec 3 niveaux énergétiques $\left(400,600\right.$ et $\left.800 \mu \mathrm{w} / \mathrm{cm}^{2}\right)$ et 4 photopériodes (6, 12,18 et 24 h d'éclairement quotidien).

\section{Mesure de la croissance mycélienne et de la pycniogénèse}

L'appréciation de la croissance mycélienne est réalisée par la mesure du diamètre des colonies, aux $5^{\mathrm{e}}$, $9^{e}$ et $14^{\mathrm{e}} \mathrm{j}$ de croissance. En même temps, la pigmentation du thalle est notée.

La quantification de la pycniogénèse est réalisée par comptage des pycnides et des pycniospores.

\section{Comptage des pycnides}

Les pycnides sont comptées après $12 \mathrm{j}$ de culture. Pour cela, des prélèvements à l'emporte pièce de 4 $\mathrm{mm}$ de diamètre, répartis sur l'ensemble de la boîte sont effectués et les fructifications sont dénombrées au microscope. Ceci permet de distinguer les pycnides fertiles, stériles et les micropycnides. Les valeurs sont exprimées par $\mathrm{cm}^{2}$ de surface du milieu de culture. Dans l'étude de l'influence du rapport $\mathrm{C} / \mathrm{N}$, des comptages précis n'ont pu être réalisés, mais une note a été attribuée suivant l'importance de la pycniogénèse $(0:$ absence de pycnides, $1:$ quelques pycnides, 2 : peu abondantes, 3 : moyennement abondantes, 4 : abondantes et 5 : très abondantes). 


\section{Comptage des pycniospores}

Lorsque l'émission des cirrhes est régulière sur l'ensemble des cultures, $10 \mathrm{ml}$ d'eau stérile sont ajoutés dans chaque boîte et un grattage de la surface gélosée est effectué avec un étaleur en crosse pour recueillir les pycniospores. La concentration des pycniospores dans la suspension obtenue est évaluée par un dénombrement sur un compte-cellules de Malassez et exprimée en spores $/ \mathrm{ml}$.

Dix répétitions sont réalisées pour chaque condition expérimentale. Après analyse de variance, les résultats sont soumis au test de Newman-Keuls, des lettres sont alors attribuées. Dans les tableaux, les valeurs suivies de lettres différentes sont significativement différentes au seuil de $5 \%$. Lorsque des observations sont réalisées sur les pycnides fertiles et stériles, l'analyse statistique est faite séparément pour ces deux types de fructifications.

\section{RÉSULTATS}

\section{Influence des variations du rapport $C / N$ sur les phénotypes mycéliens}

Des variations des phénotypes mycéliens peuvent être observées sur chaque souche suivant les valeurs des rapports $\mathrm{C} / \mathrm{N}$. Un rapport $\mathrm{C} / \mathrm{N}=$ 10 induit un mycélium blanchâtre, alors qu'un rapport $\mathrm{C} / \mathrm{N}=20$ induit une coloration blanc-rosé dumycélium. Des rapports $\mathrm{C} / \mathrm{N} \geq 80$ provoquent un développement de colonies de couleur verte devenant de plus en plus sombre avec l'élévation de la valeur de ce rapport $\mathrm{C} / \mathrm{N}$, et ceci, indépendamment des valeurs absolues en carbone et en azote.

\section{Influence des variations du rapport $\mathrm{C} / \mathrm{N}$ sur la pycniogénèse}

Les productions de pycnides et de pycniospores ont été étudiées sur une culture de souche mono-ascosporée (A 3.5) maintenue à $14{ }^{\circ} \mathrm{C}$ sous un éclairement de $12 \mathrm{~h}$ par $24 \mathrm{~h}$ avec un niveau énergétique de $700 \mu \mathrm{w} / \mathrm{cm}^{2}$.

Les résultats expérimentaux (tableau I) mettent en valeur l'importance du rapport $\mathrm{C} / \mathrm{N}$ sur la pycniogénèse. Les rapports $\mathrm{C} / \mathrm{N}<40$ permettent une production optimale de pycniospores. L'influence de ce rapport se manifeste surtout sur l'émission des conidies et non sur le nombre de pycnides, en effet, des rapports $>40(60,80$ ou $160)$ permettent une bonne production de pyc- nides mais avec une émission de spores réduite. Pour les rapports $\mathrm{C} / \mathrm{N}$ élevés, la très faible émission des cirrhes peut s'expliquer par un développement des fructifications à l'intérieur du milieu gélosé.

Tableau I. Étude de l'influence des variations des concentrations en carbone (galactose) et en azote (glycine) dans le milieu synthétique (S), sur la pycniogénèse de $P$ nodorum.

\begin{tabular}{|c|c|c|c|c|}
\hline \multicolumn{2}{|c|}{$\begin{array}{c}\text { Concentration } \\
(m g / l)\end{array}$} & \multirow[t]{2}{*}{$\begin{array}{l}\text { Rapport } \\
C / N\end{array}$} & \multicolumn{2}{|c|}{ Production } \\
\hline$C$ & $N$ & & Pycnides & $\begin{array}{c}\text { Pycniospores } \\
\left(\times 10^{6} / \mathrm{ml}\right)\end{array}$ \\
\hline \multirow[t]{5}{*}{400} & 25 & 16 & $3^{1}$ & $1,34 \mathrm{gh}$ \\
\hline & 50 & 8 & 3 & $1,28 \mathrm{gh}$ \\
\hline & 100 & 4 & 3 & $1,29 \mathrm{gh}$ \\
\hline & 200 & 2 & 2 & $0,18^{h}$ \\
\hline & 400 & 1 & 3 & $3,70^{f}$ \\
\hline \multirow[t]{5}{*}{1000} & 25 & 40 & 4 & $0,24^{h}$ \\
\hline & 50 & 20 & 3 & $1,96 \mathrm{~g}$ \\
\hline & 100 & 10 & 3 & $9,45^{b}$ \\
\hline & 200 & 5 & 3 & $6,82^{d}$ \\
\hline & 400 & 2,5 & 3 & $3,81+$ \\
\hline \multirow[t]{5}{*}{2000} & 25 & 80 & 4 & $0,22^{h}$ \\
\hline & 50 & 40 & 4 & $0,33^{h}$ \\
\hline & 100 & 20 & 4 & $7,25^{d}$ \\
\hline & 200 & 10 & 4 & 6,42 de \\
\hline & 400 & 5 & 4 & $8,85 b c$ \\
\hline \multirow[t]{5}{*}{4000} & 25 & 160 & 4 & $0,06^{h}$ \\
\hline & 50 & 80 & 4 & 0 \\
\hline & 100 & 80 & 4 & $0,13^{n}$ \\
\hline & 200 & 20 & 5 & $5,76^{e}$ \\
\hline & 400 & 10 & 5 & $12,12^{a}$ \\
\hline \multirow[t]{4}{*}{6000} & 50 & 120 & 4 & $0,41^{h}$ \\
\hline & 100 & 60 & 5 & $0,16^{h}$ \\
\hline & 200 & 30 & 5 & $8,49^{c}$ \\
\hline & 400 & 15 & 3 & $7,10^{d}$ \\
\hline \multirow[t]{4}{*}{8000} & 50 & 160 & 4 & $0,03 \mathrm{~h}$ \\
\hline & 100 & 80 & 4 & 0 \\
\hline & 200 & 40 & 4 & $0,24 \mathrm{~h}$ \\
\hline & 400 & 20 & 5 & 6,46 de \\
\hline \multirow[t]{3}{*}{16000} & 100 & 160 & 4 & 0 \\
\hline & 200 & 80 & 4 & $0,33^{h}$ \\
\hline & 400 & 40 & 4 & 8,77 bc \\
\hline
\end{tabular}

\footnotetext{
${ }^{1}$ La quantité des pycnides est notée suivant une échelle de 0 à 5 . Les valeurs suivies de la même lettre en exposant ne sont pas significativement différentes.
} 
Le milieu le plus favorable pour la production et l'émission de pycniospores contient $4000 \mathrm{mg} / \mathrm{l}$ de $\mathrm{C}$ et $400 \mathrm{mg} / \mathrm{l}$ de $\mathrm{N}$ soit un rapport $\mathrm{C} / \mathrm{N}$ de 10 . Ce milieu désigné sous le nom de milieu $\mathrm{S}$ a été comparé au milieu de Richards (1951) et au milieu semi-synthétique Czapek dox-V8 (Cooke et Gareth-Jones, 1970). Les cultures sont réalisées à $14{ }^{\circ} \mathrm{C}$ dans les mêmes conditions d'éclairement que précédemment.

Les résultats relatifs à la croissance mycélienne et à la production de pycniospores sont reportés dans le tableau II. La croissance mycélienne après $14 \mathrm{j}$ de culture sur le milieu $\mathrm{S}$ $(6,63 \mathrm{~cm})$ est significativement inférieure à celle ob-servée sur les milieux de Richards $(7,13 \mathrm{~cm})$ et Czapek Dox-V8 $(7,01 \mathrm{~cm})$. Par contre, la production de pycniospores est plus importante dans le cas du milieu $S$ que nous proposons.

\section{Influence de la température et de la lumière sur la conidiogénèse de souches mono-ascosporées}

Dans le but de définir les conditions optimales de la pycniogénèse sur le milieu $S$ et de vérifier si des exigences différentes pouvaient apparaître entre des souches mono-ascosporées nous avons réalisé cette étude pour les souches A $2.3(+)$ et A $2.6(-)$.

\section{Influence de la température}

Les résultats relatifs à l'étude de l'influence de la température sont reportés dans le tableau III. Pour les 2 souches, la production de pycnides fertiles est plus importante à la température de $14{ }^{\circ} \mathrm{C}\left(531,0\right.$ pycnides $/ \mathrm{cm}^{2}$ pour A 2.6 et 316,6 pycnides $/ \mathrm{cm}^{2}$ pour A 2.3). Mis à part un potentiel de production de pycnides plus important à 10 ${ }^{\circ} \mathrm{C}$ et à $14{ }^{\circ} \mathrm{C}$ pour la souche $\mathrm{A} 2.6$, aucune différence d'exigence vis-à-vis du facteur température ne se manifeste entre les 2 souches. Aux températures de $18{ }^{\circ} \mathrm{C}$ et $24^{\circ} \mathrm{C}$, des quantités plus élevées de petites pycnides stériles ou peu fertiles sont observées, une faible quantité poursuit son développement jusqu'au stade permettant la conidiogenèse.

La température de $14{ }^{\circ} \mathrm{C}$, la plus favorable pour la macropycniogenèse, a été retenue pour la suite de ce travail.

\section{Influence de la lumière}

L'influence de la lumière sur la formation des macropycnides a été étudiée en fonction de la durée journalière et de la valeur d'éclairement.

\section{Influence de la durée journalière de l'éclairement}

La production de pycnides fertiles obtenues selon diverses valeurs d'éclairement quotidien $(0,6,12,18$ et $24 \mathrm{~h})$ est reportée dans le tableau IV. Un éclairement quotidien de $12 \mathrm{~h}$ assure une

Tableau II. Comparaison de la pycniogénèse et du développement mycélien de $P$ nodorum obtenus sur le milieu $S$ par rapport à 2 milieux de référence (Richard et Czapek Dox V8). $14{ }^{\circ} \mathrm{C}$, éclairement quotidien de $12 \mathrm{~h}$ en lumière noire.

\begin{tabular}{|c|c|c|c|c|}
\hline \multirow[t]{2}{*}{ Milieu } & \multicolumn{3}{|c|}{$\begin{array}{l}\text { Croissance } \\
\text { mycélienne } \\
\text { (cm) }\end{array}$} & \multirow{2}{*}{$\begin{array}{l}\text { Production de } \\
\text { pycniospores } \\
\frac{\left(\times 10^{6} / \mathrm{ml}\right)}{17^{e} j}\end{array}$} \\
\hline & $5^{e j}$ & $g^{e j}$ & $14^{e} j$ & \\
\hline Milieu S & $2,22^{c}$ & $4,52 \mathrm{~b}$ & $6,63^{b}$ & $12,84 a$ \\
\hline Richards & $2,46^{b}$ & $4,83^{a}$ & $7,13^{a}$ & $3,45^{c}$ \\
\hline Czapek Dox V8 & $2,71^{a}$ & $4,78^{a}$ & $7,01^{a}$ & $8,70^{b}$ \\
\hline
\end{tabular}

${ }^{1}$ Croissance en diamètre.

Tableau III. Influence de la température de croissance sur la production de pycnides (fertiles et stériles) de $P$ nodorum chez deux souches mono-ascosporées (A 2.3 et $A$ 2.6).

\begin{tabular}{|c|c|c|c|c|}
\hline \multirow[t]{2}{*}{$\begin{array}{l}\text { Températur } \\
\text { de croissan }\end{array}$} & \multicolumn{2}{|c|}{$\begin{array}{c}\text { Pycnides } \\
\text { fertiles } / \mathrm{cm}^{2}\end{array}$} & \multicolumn{2}{|c|}{$\begin{array}{c}\text { Pycnides } \\
\text { stériles } / \mathrm{cm}^{2}\end{array}$} \\
\hline & A $2.3(+)$ & A $2.6(-)$ & $A 2.3(+)$ & $A 2.6(-)$ \\
\hline $10^{\circ} \mathrm{C}$ & $255,5 d$ & $466,9^{b}$ & $17,0^{f}$ & $202,4^{d}$ \\
\hline $14{ }^{\circ} \mathrm{C}$ & $316,6^{c}$ & $531,0^{a}$ & $24,9^{f}$ & $112,9^{e}$ \\
\hline $18^{\circ} \mathrm{C}$ & $45,7^{e}$ & $41,4^{e}$ & $588,8^{b}$ & $754,4^{a}$ \\
\hline $24^{\circ} \mathrm{C}$ & $23,9 \mathrm{e}$ & $33,6^{\mathrm{e}}$ & $796,7^{\text {a }}$ & $307,5^{c}$ \\
\hline
\end{tabular}

Culture sur le milieu $S$ sous un éclairement en lumière noire $(12 / 12$ environ $600 \mu \mathrm{w} / \mathrm{cm}^{2}$ ). 
meilleure production de pycnides. La souche A2.6 présente une potentialité plus importante $\left(335,6\right.$ pycnides $\left./ \mathrm{cm}^{2}\right)$ que la souche A2.3 $(242.3$ pycnides $/ \mathrm{cm}^{2}$ ) pour $12 \mathrm{~h}$ d'éclairement quotidien. Pour les cultures maintenues à l'obscurité, il n'y a pas de pycniogenèse.

\section{Influence de la valeur de l'éclairement}

Quatre types d'éclairement $(400,600$ et $800 \mu \mathrm{w} /$ $\mathrm{cm}^{2}$ ) ont été utilisés sur des cultures recevant un éclairement quotidien de $12 \mathrm{~h}$ à la température de $14{ }^{\circ} \mathrm{C}$. Le tableau $V$ met en évidence pour la souche A2.3 un optimum de production de pycnides pour une valeur de $600 \mu \mathrm{w} / \mathrm{cm}^{2}$ (193,0 pycnides $/ \mathrm{cm}^{2}$ ), alors que la souche A2.6 présente une meilleure production sur une plage plus étendue allant de $400 \mu \mathrm{w} / \mathrm{cm}^{2}\left(403,1\right.$ pycnides $\left./ \mathrm{cm}^{2}\right)$ à $600 \mu \mathrm{w} / \mathrm{cm}^{2}\left(455,1\right.$ pycnides $\left./ \mathrm{cm}^{2}\right)$.

Tableau IV. Influence de la durée de l'éclairement sur la production de pycnides (fertiles et stériles) de $P$ nodorum chez 2 souches mono-ascosporées (A 2.3 et $A$ 2.6).

\begin{tabular}{|c|c|c|c|c|}
\hline \multirow[t]{2}{*}{$\begin{array}{l}\text { Durée de } \\
\text { l'éclaireme }\end{array}$} & \multicolumn{2}{|c|}{$\begin{array}{c}\text { Pycnides } \\
\text { fertiles } / \mathrm{cm}^{2}\end{array}$} & \multicolumn{2}{|c|}{$\begin{array}{c}\text { Pycnides } \\
\text { stériles } / \mathrm{cm}^{2}\end{array}$} \\
\hline & A $2.3(+)$ & A $2.6(-)$ & $A 2.3(t)$ & $A 2.6(-)$ \\
\hline $0 / 24^{1}$ & 0 & 0 & 0 & 0 \\
\hline $6 / 18$ & $217,5^{c}$ & $243,4^{b}$ & $98,9 \mathrm{c}$ & 300,2 a \\
\hline $12 / 12$ & $242,3^{b}$ & $335,6^{a}$ & $110,2^{c}$ & $242,0^{b}$ \\
\hline $18 / 6$ & $106,2^{e}$ & $139,3^{d}$ & $221,6^{\mathrm{b}}$ & $150,7^{c}$ \\
\hline $24 / 0$ & 106,1 e & $107,9^{e}$ & $245,0^{b}$ & $114,1^{c}$ \\
\hline
\end{tabular}

\footnotetext{
1 La première valeur indique le nombre d'heures correspondant a la période éclairée; culture sur le milieu $\mathrm{S}$ sous un éclairement en lumière noire (environ $600 \mu \mathrm{w} / \mathrm{cm} 2$ ) à $14{ }^{\circ} \mathrm{C}$.
}

Tableau V. Influence de la valeur de l'éclairement sur la production de pycnides de $P$ nodorum chez deux souches monoascosporées (A 2.3 et A 2.6).

\begin{tabular}{ccc}
\hline \multirow{2}{*}{$\begin{array}{c}\text { Intensité de } \\
\text { l'éclairement } \\
\left(\mu \mathrm{w} / \mathrm{cm}^{2}\right)\end{array}$} & \multicolumn{2}{c}{ Souches } \\
\cline { 2 - 3 } & $A 2.3(+)$ & $A 2.6(-)$ \\
\hline & & \\
400 & $129,0^{1 \mathrm{c}}$ & $403,1^{\mathrm{a}}$ \\
600 & $193,0^{\mathrm{b}}$ & $455,1^{\mathrm{a}}$ \\
800 & $73,9^{\mathrm{C}}$ & $248,8^{\mathrm{b}}$ \\
\hline
\end{tabular}

\footnotetext{
1 Nombre de pycnides $/ \mathrm{cm}^{2}$. Culture sur le milieu $S$ sous un éclairement en lumière noire à $14^{\circ} \mathrm{C}$.
}

\section{DISCUSSION ET CONCLUSION}

Le galactose et la glycine ont été retenus comme source carbonée et azotée du milieu $\mathrm{S}$, à la suite du travail de Richards (1951) qui avait obtenu une conidiogenèse plus abondante avec ces composés.

L'étude du rapport $\mathrm{C} / \mathrm{N}$ a montré l'importance de ce facteur sur la production de pycnides et surtout sur la production des pycniospores. L'influence du rapport $\mathrm{C} / \mathrm{N}$ sur la croissance végétative, la conidiogenèse et le développement sexué avait déjà été soulignée par différents auteurs. Ainsi chez Gnomonia leptostyla (Fayret, 1975), des valeurs optimales très différentes orientent la morphogenèse soit vers la croissance mycélienne et la conidiogenèse $(\mathrm{C} / \mathrm{N}=13$ et 23$)$, soit vers la morphogenèse périthéciale $(C / N=83)$. La conidiogenèse de $S$ nodorum est optimale pour un rapport $\mathrm{C} / \mathrm{N}=10$. Notons toutefois qu'avec le milieu de Dehorter (1985) et avec une gamme de rapports $\mathrm{C} / \mathrm{N}$ très étendue, nous n'avons pas obtenu de périthèces (Halama, 1991).

L'étude des facteurs physiques permet de définir une température optimale de $14{ }^{\circ} \mathrm{C}$ pour la pycniogénèse de $S$ nodorum; ces résultats divergent de ceux de Richard (1951) qui obtient la sporulation de $S$ nodorum uniquement à $20^{\circ} \mathrm{C}$. Par contre, ils sont proches de ceux de Calpouzos et Lapis (1970) qui observent une meilleure sporulation à $15^{\circ} \mathrm{C}$. Nous avons observé une initiation importante des pycnides à $18^{\circ} \mathrm{C}$, cependant peu d'entre elles poursuivent leur développement jusqu'au stade permettant la formation des conidies. Ce comportement présente une certaine analogie avec celui observé chez certaines espèces du genre Alternaria pour lesquelles la phase d'induction de la conidiogenèse est stimulée par des températures élevées alors que la phase terminale permettant la formation des conidiospores est favorisée par des températures basses (Leach, 1967a).

La photo-induction de la pycniogenèse chez $S$ nodorum a déjà été décrite par Calpouzos et Lapis (1970), Cooke et Gareth-Jones (1970). Une photopériode de $12 \mathrm{~h}$ permet la meilleure production de pycnides, ce résultat rejoint l'observation faite par Aita et Neto (1979). Un éclairement continu permet la sporulation de $S$ nodorum, cette condition a également été utilisée chez cette espèce par Richards (1951), Leach (1967b), Cooke et Gareth-Jones (1970). La lumière continue avec un éclairement énergétique de $600 \mu \mathrm{w} / \mathrm{cm}^{2}$ a un effet dépressif sur les 2 
souches utilisées. En ce qui concerne la valeur de l'éclairement énergétique, Richards (1951) avait constaté qu'une sporulation abondante de $S$ nodorum intervenait pour des éclairements atteignant $200 \mu \mathrm{w} / \mathrm{cm}^{2}$, mais c'était la valeur maximale expérimentée par cet auteur. Calpouzos et Lapis (1970) en étudiant une échelle de valeurs plus étendue (de $8-800 \mu \mathrm{w} / \mathrm{cm}^{2}$ ) ont constaté qu'un isolat monoconidien présentait un optimum vers $600 \mu \mathrm{w} / \mathrm{cm}^{2}$ alors que pour l'autre isolat, il se situait entre 400 et $800 \mu \mathrm{w} / \mathrm{cm}^{2}$. Nous observons également une différence de comportement entre les souches utilisées, l'une ayant une exigence plus stricte vis-à-vis de l'éclairement énergétique $\left(600 \mu \mathrm{w} / \mathrm{cm}^{2}\right.$ pour $A$ 2.3) alors que l'autre présente un optimum plus large (400 et $600 \mu \mathrm{w} / \mathrm{cm}^{2}$ ). Cependant, la valeur optimale de $600 \mu \mathrm{w} / \mathrm{cm}^{2}$ est commune pour les 2 souches.

En conclusion, nous avons optimisé et stabilisé la pycniogénèse de 2 souches de compatibilités sexuelles opposées en modifiant le milieu:

- par un apport plus étendu d'éléments minéraux, surtout de microéléments dont l'influence spécifique devrait être ultérieurement précisée;

- en déterminant le rapport $\mathrm{C} / \mathrm{N}=10 \mathrm{comme}$ optimal pour la pycniogénèse, le galactose et la glycine constituant les sources de carbone et d'azote.

Ce travail conduit à préciser la plage optimale de production de conidies en fonction des facteurs physiques : une température de $14{ }^{\circ} \mathrm{C}$ et un éclairement quotidien de $12 \mathrm{~h}$ en lumière noire avec un niveau énergétique de $600 \mu \mathrm{w} / \mathrm{cm}^{2}$. II faut noter que vis-à-vis de la température et de la durée quotidienne de l'éclairement, les 2 souches utilisées ne présentent pas de différences, seule une potentialité plus importante apparaît pour l'une d'entre elles (A 2.6) qui présente toujours une sporulation plus importante quel que soit le facteur physique étudié. Cette observation confirme des observations précédentes où des potentialités différentes de sporulation apparaîssaient entre les 8 souches monoascosporées issues d'un même asque (Halama et Lacoste, 1991).

Pour le maintien des facultés conidiogènes, outre ces conditions optimales, il faut signaler la nécessité d'un repiquage fréquent des cultures sporulantes (environ tous les $10 \mathrm{j}$ ).

La détermination des conditions optimales permettra la production de pycniospores, chez des souches de signe sexuel complémentaire et la réalisation de contaminations en plein champ avec un matériel défini par rapport au signe sexuel. Par ailleurs, cette optimisation de la coni- diogenèse et sa stabilité au cours des repiquages successifs pour des souches monoascosporées de $P$ nodorum permettra d'aborder l'étude de l'hérédité du pouvoir pathogène par la réalisation de croisements puis l'appréciation de l'agressivité de la descendance.

\section{RÉFÉRENCES}

Aita L, Neto JP, Da C (1979) Effect of light conditions on sporulation of three isolates of Septoria nodorum Berck. Agron Subriograndense 15, 173-178

Alligham EA, Jackson LF (1981) Variation in pathogenicity, virulence and aggressiveness of Septoria nodorum in Florida. Phytopathology 71, 1080-1085

Calpouzos L, Lapis DB (1970) Effects of light on pycnidium formation, sporulation, and tropism by Septoria nodorum. Phytopathology 60, 791-794

Cooke BM, Gareth-Jones D (1970) The effect of near ultraviolet irradiation of Septoria nodorum and $S$ tritici. Trans Br Mycol Soc 54, 221-226

Dehorter B (1985) Déterminisme et physiologie de la reproduction sexuée du Nectria galligena Bres. Thèse Doct sci nat Lille, $240 p$

Fayret J (1975) Étude du cycle de reproduction de Gnomonia leptostyla (Fr) Ces et de Not. Déterminisme et physiologie. Thèse Doct sci nat, Univ Paul Sabatier Toulouse III, $296 p$

Griffiths E, Ao HC (1980) Variation in Septoria nodorum. Ann Appl Biol 94, 294-296

Halama P (1991) Phaeosphaeria nodorum (ex Leptosphaeria nodorum) téléomorphe de Septoria nodorum. Déterminisme et ontogénie-hérédité du pouvoir pathogène. Thèse Doct Univ, Univ Lille

Halama P, Lacoste L (1991) Déterminisme de la reproduction sexuée de Phaeosphaeria (Leptosphaeria) nodorum, agent de la septoriose du blé. I. Hétérothallisme et rôle des microspores. Can J Bot 69, 95-99

Leach CM (1967a) Interaction of near ultraviolet light and temperature on sporulation of the fungi Alternaria, Cercosporella, Fusarium, Helminthosporium and Stemphylium. Can J Bot 45, 1999-2016

Lacoste L (1965) Biologie naturelie et culturale du genre Leptosphaeria Cesati et Notaris. Déterminisme de la reproduction sexuelle. Thèse doc Sci nat Toulouse, $234 p$

Leach CM (1967b) The light factor in the detection and identification of seed borne fungi. Proc Int Seed Testing Ass 32, 565-589

Morquer R (1931) Recherches morphogéniques sur le Dactylium macrosporum. Thèse Fac Sci Paris

Richards GS (1951) Factors influencing sporulation by Septoria nodorum. Phytopathology 41, 571-578

Scharen AL, Krupinsky JM (1970) Cultural and inoculation studies of Septoria nodorum, cause of glume blotch of wheat. Phytopathology 60, 1480-1485 